\title{
Performance of the fuel injector in supersonic combustor
}

\author{
Zhe Wei ${ }^{1}$, LiHong Chen ${ }^{2}$, Hongbin $\mathrm{Gu}^{3}, \mathrm{Fei}^{4}{ }^{4}$, Shenglong $\mathrm{Gu}^{5}$, XinYu Chang ${ }^{6}$ \\ Key Lab of High Temperature Gas Dynamics, \\ Institute of Mechanics, Chinese Academy of Sciences, Beijing 100190, P.R. China
}

Enhancing the fuel-air mixing is critical for scramjet combustor performance. The performance of four different aerodynamic ramp injectors was reported in this paper. The experiments were conducted in a direct-connected scramjet test facility. The concentration profiles were obtained by gas sampling and chromatogram analysis. The pictures of the flow field were obtained by using laser scatter. It would be used to analyze the flow field generated by the aerodynamic ramp, then optimize the layout of the injectors. The results would offer some useful information for engine design.

\section{Introduction}

CCRAMJET is the key technology for the hypersonic aircraft. Improving the fuel-air mixing is a critical and challenge for scramjet combustor performance because air travels through the combustor in a high velocity which makes the fuel stay in the combustor for only few milliseconds.

The methods of enhancing the fuel-air mixing can be divided into two kinds: passive and active. The passive methods operate through changing the structure of the flow fields, forming the vortices and so on. It includes physical ramp, aerodynamic ramp, cave in the wall, pylon, and so on. The active method is using active force to inspirit the flow field, such as impulse injection.

An aerodynamic ramp is actually an array of injectors. Compared with a physical ramp, it could provide certain advantageous flow features like streamwise vorticity and adequate fuel penetration of a physical ramp, while improving the fuel-air mixing, total pressure recovery and avoiding burning down the intrusive physical ramp. Fig. 1 is a typical flow field of an aerodynamic ramp with 1-hole injector.

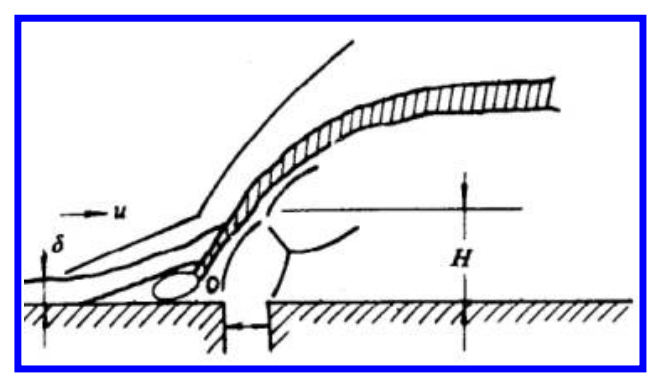

Fig.1 Sketch of 1-hole injector

\footnotetext{
${ }^{1}$ Graduate Student,

2 Professor,

3 Associate Professor,

4 Assistant Professor,

5 Ph.D Student,

6 Professor,
}

Key lab of high temperature gas dynamics, Key lab of high temperature gas dynamics, Key lab of high temperature gas dynamics, Key lab of high temperature gas dynamics, Key lab of high temperature gas dynamics, Key lab of high temperature gas dynamics, weizhejiji@yahoo.com.cn

lhchen@imech.acn

guhb@imech.ac.cn

lifei@imech.ac.cn

gushenglong@imech.ac.cn

xychang@imech.acn 


\section{Test Facility}

The effect of aerodynamic ramp on supersonic mixing was investigated in this paper. The experiments were conducted in the direct-connected scramjet test facility with Mach 3. The gas of carbon dioxide was injected though sonic nozzles into the main flow. The flow field was illustrated by laser scatter, and the concentration profiles were obtained by gas sampling and chromatogram analysis.

\section{2-1 Direct-connected scramjet test facility}

The direct-connected scramjet test facility was using the method of burning hydrogen and adding oxygen to create the main flow with high temperature and high pressure. The main flow was Mach 3 with total temperature $1000 \mathrm{~K}$ and total pressure $2 \mathrm{MPa}$. Fig.2 was the schematic of the facility where flow was from left to right. It consisted of supersonic nozzle, isolator, combustor 1 and combustor 2 . The combustors had removable inserts on all four walls allowing optical access, installation of instrumentations and a wide parametric design margin for fuel injections.

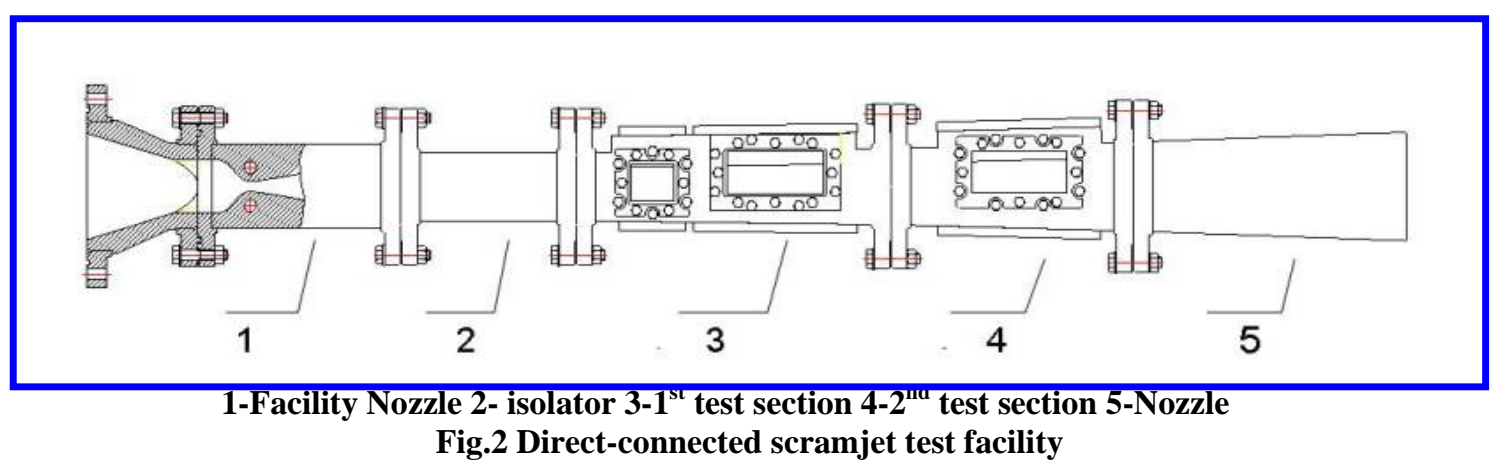

\section{2-2 Aerodynamic ramp nozzle}

The gas of carbon dioxide was injected from the wall in a sonic velocity into the main flow. Four different aerodynamic nozzles were designed and experimented. They all had the same effective diameter which was $2.4 \mathrm{~mm}$ and their patters were showed as follows:
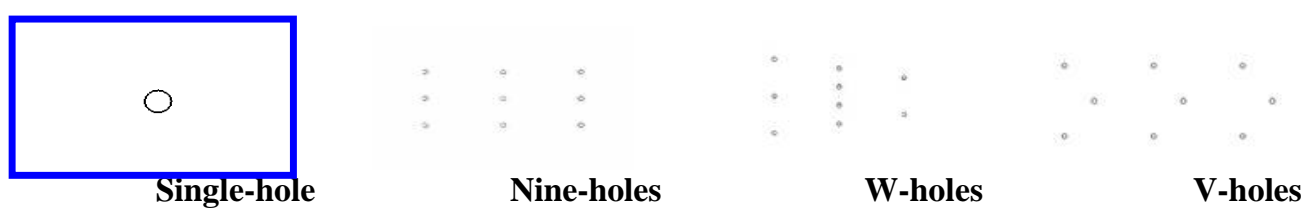

Fig 3 Scheme of different nozzles

\section{2-3 Measurements}

A sampling system was used to determine the concentration of carbon dioxide in the downstream of the flow filed. The probe was on the symmetrical plane, and $70 \mathrm{~mm}$ downstram from the $\mathrm{CO}_{2}$ injection. There were four sampling points with the distance of $5,10,15$, and $25 \mathrm{~mm}$ from the wall respectively, showed in Fig 4 . The effect of mixing would be evaluated from the $\mathrm{CO}_{2}$ distribution. In addition, through adding particles to the injection flow and using the laser scatter, the clear picture would be got to reveal the mixing between the coming flow and injecting flow, which would be helpful to analyze the structure of the flow field. 


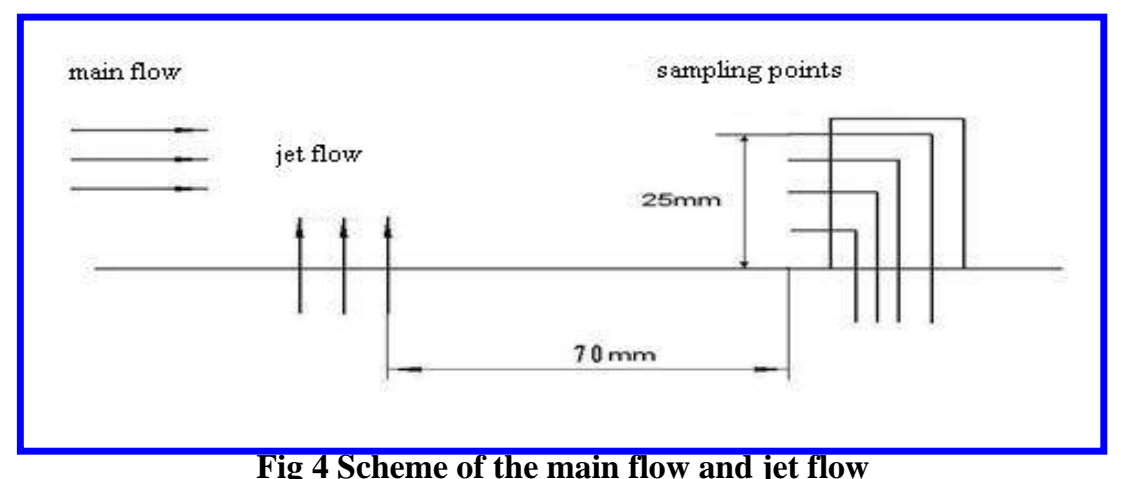

Fig 4 Scheme of the main flow and jet flow

\section{Results and Analysis}

\section{3-1 Concentration analysis}

The $\mathrm{CO}_{2}$ distributions were obtained. Fig 5, Fig 6, Fig 7 and Fig 8 were the concentration distributions of four kinds of nozzles under two different pressures which were $2 \mathrm{MPa}$ and $3 \mathrm{MPa}$. The distances between the wall and the four sampling points were $25 \mathrm{~mm}, 15 \mathrm{~mm}, 10 \mathrm{~mm}$ and $5 \mathrm{~mm}$ respectively. The datum showed in the picture were the relative concentration results, which were obtained from the original datum divided by the ideal concentration. The ideal concentration means the uniform value of the $\mathrm{CO}_{2}$ to the global main flow. Thus the closer the datum to number 1 , the better the mixing effect.

From the four pictures, we could get the results: the concentrations gotten by the four sampling points of the four kinds of nozzles were larger than the ideal concentration. So the concentrations of carbon dioxide in other places were lower than the ideal datum which showed the mixing between the injection flow and the main flow was not developed well. This was the exactly challenge for scramjet combustor.

From the four pictures, we could find that as the pressure increased from $2 \mathrm{MPa}$ to $3 \mathrm{MPa}$, the relative concentration of all the four kinds of injection nozzle trended to be uniform, in which the difference between the peak and bottom value became smaller. The reason would be the increasing pressure made the jet momentum larger which would enhance the mixing of the injection flow and the main flow. However, the $\mathrm{CO}_{2}$ profiles didn't change a lot, in which the relative concentrations of carbon dioxide were becoming larger as they were closed to the wall, and the largest concentration was at the place of about $10 \mathrm{~mm}$ from the wall. Therefore, the effect of the jet pressure on mixing was limited, and it didn't change the flow pattern of the jet penetration

Fig 9 and Fig 10 were the comparing results of four kinds of nozzles under two pressures. Both the two figs showed that the relative concentrations of single-hole nozzle were more uniform than the other three kinds of nozzles, especially in the distance of $15 \mathrm{~mm}$ to $5 \mathrm{~mm}$. That meant carbon dioxide coming from single-hole congregated in the axial direction and made better mixing due to the focus injection. However, the spanwise mixing should be measured further to evaluate the global effect.

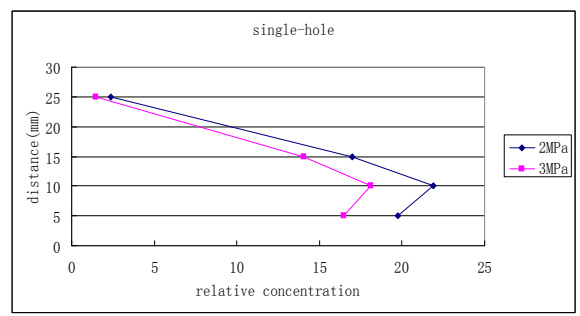

Fig 5 Relative concentrations of Single-hole

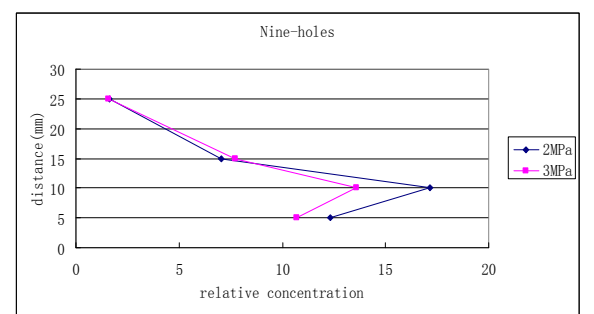

Fig 6 Relative concentrations of Nine-holes 


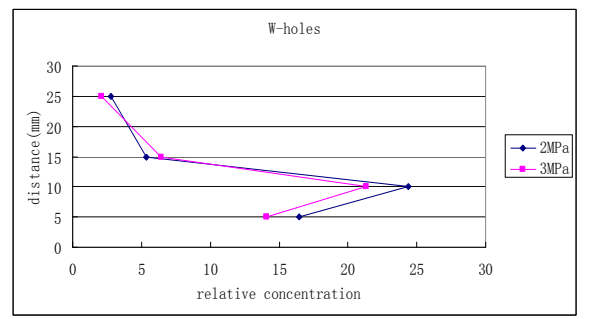

Fig 7 Relative concentrations of W-holes

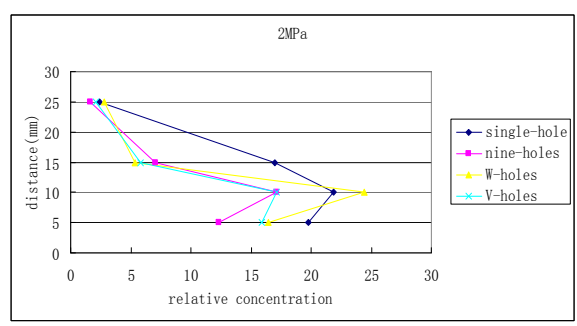

Fig 9 Relative concentrations under $2 \mathrm{MPa}$

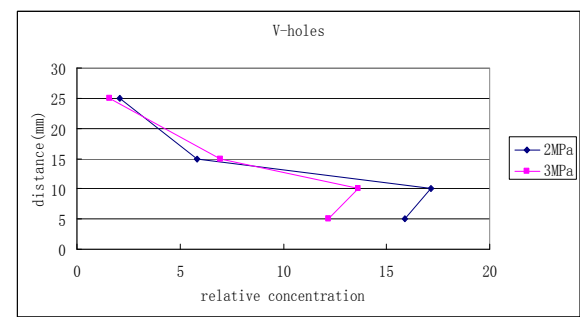

Fig 8 Relative concentrations of V-holes

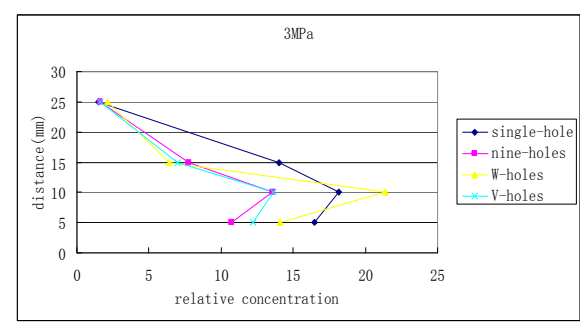

Fig 10 Relative concentrations under $3 \mathrm{MPa}$

\section{3-2 Scatter picture}

In order to got the flow structure, the laser scatter was used on the central symmetrical plane of the injection holes. Fig 11 were the scatter pictures: (A) was the subsonic jet of one hole into atmosphere; (B) was the sonic jet of one hole into atmosphere; (C) was the sonic jet of one hole to the supersonic main flow with Mach 3; (D) was the sonic jet of Nine-holes to the supersonic main flow. Fig.11 (A) shown the structure of large scale vortices which was the characteristic of the subsonic jet. Especially in the end of the jet, the structures of large scale vortices were very clear. Fig.11 (B) shown the structures of large scales disappeared for the sonic jet. The jet flow field was a cone type and the jet area augmented as the jet distance increased. Fig.11(C) was the sonic jet went into the supersonic main flow, that the cone type of jet flow field was destroyed by the supersonic main flow. The jet flow spread to the core of the main flow as it went to the downstream. During the process, some vortices were formed and they interacted with the shear layer which made the jet flow gradually mix with the main flow. Fig.11(D) was the flow field of the sonic jet of nine holes to the supersonic main flow. The picture was on the symmetrical plane through the central three holes. From Fig.11(D) we could find the effect of aerodynamic ramp clearly. Owing to the block effect of the former hole to the main flow, the latter hole had a stronger injection and deeper penetration than the former hole, which was exactly the character of the aerodynamic ramp. In addition, comparing (C) and (D), the penetrating depth of one hole was deeper than nine holes but particles dispersed better for nine holes. That meant the hole with bigger diameter had a bigger flow rate and had a deeper penetration. However, with the same effective diameter, nine holes had a better mixing.

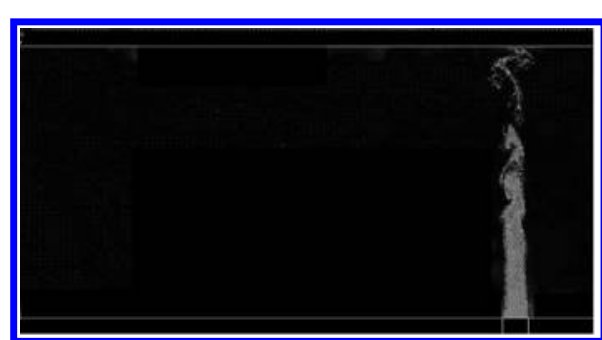

A: subsonic free jet of one hole

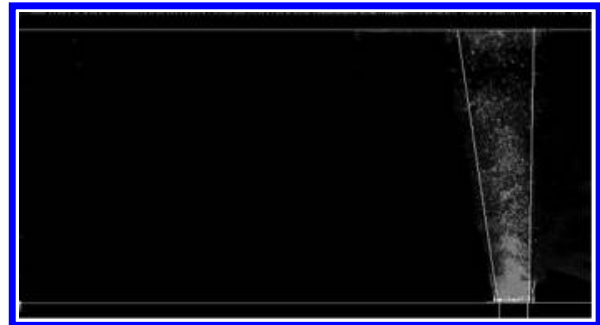

B: sonic free jet of one hole 

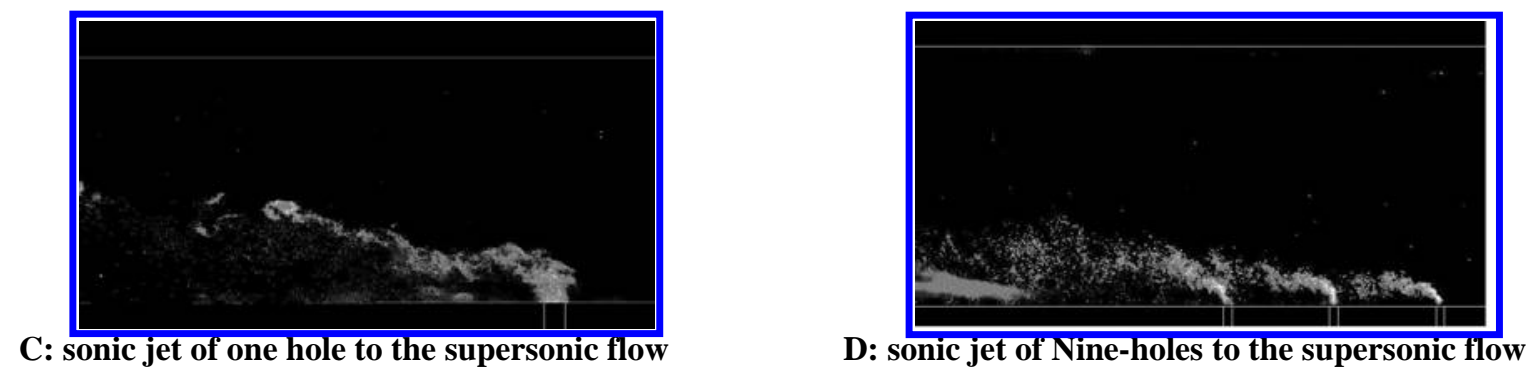

Fig 11 Scatter pictures

\section{Summary and Conclusion}

The effect of aerodynamic ramp on supersonic mixing was investigated in the direct-connected scramjet test facility with Mach 3 . The results were as follows:

(1) As the jet pressure increased from $2 \mathrm{MPa}$ to $3 \mathrm{MPa}$, the relative concentration of all the four kinds of injection nozzle trended to be uniform. This showed that the increasing pressure made the jet momentum larger which would enhance the mixing of the injection flow and the main flow.

(2) The penetrations of the jet were limited. The concentrations of carbon dioxide were becoming larger as they were closed to the wall. The largest concentration occurred at the place of about $10 \mathrm{~mm}$ from the wall.

(3) The scatter pictures shown the interaction between the jet and main flow. The effect of the aerodynamic ramp was that the owing to the block effect of the former hole to the main flow, the latter hole had a stronger injection and deeper penetration than the former hole.

\section{Acknowledgments}

The project is funded by National Natural Science Foundation of China (90305022, 10525212, 10621202).

\section{References}

[1] D.R.Eklund, M.R.Gruber. Study of a Supersonic Combustor Employing an Aerodynamic Ramp Pilot Injector, 35th AIAA/ASME/SAE/ASEE Joint Propulsion Conference \& Exhibit 1999-6-20 24, Los Angeles, California

[2] Tohru Mitani, Masahiro Takahashi, Sadatake Tomioka, Tetsuo Hiraiwa, Kouichiro Tani. Analyses and Application of Gas Sampling to Scramjet Testing. Journal of Propulsion and Power, Vol.15, No.4,1999,572 577

[3] Raymond P. Fuller, Pei-Kuan Wu, Abdollah S. Nejad, Joseph A. Schetz. Comparison of Physical and Aerodynamic Rampsas Fuel Injectors in Supersonic Flow. Journal of Propulsion and Power, Vol.14, No.2,1998,135 145 
This article has been cited by:

1. Yi Zhao, Qing Shen, Faming GuanQuasi-One-Dimensional Analysis of Supersonic Combustor Performance . [Citation] [PDF] [PDF Plus]

2. Lu Tian, Lihong Chen, Qiang Chen, Fei Li, Xinyu Chang. 2014. Quasi-One-Dimensional Multimodes Analysis for DualMode Scramjet. Journal of Propulsion and Power 30:6, 1559-1567. [Abstract] [Full Text] [PDF] [PDF Plus] 